\title{
ON THE ORNESS OF SUOWA OPERATORS
}

\author{
Bonifacio Llamazares
}

Dpto. de Economía Aplicada, Instituto de Matemáticas (IMUVA), Universidad de Valladolid, Avda. Valle de Esgueva 6, 47011 Valladolid, Spain

Email: boni@eco.uva.es

\begin{abstract}
There is in the literature a great variety of functions utilized in the aggregation processes. For this reason, numerous indicators have been suggested to understand the behavior of such functions. One of the measures proposed for this purpose is the orness, which allows to know the degree of closeness to the maximum. The aim of this paper is to provide the orness of some specific cases of SUOWA operators, a family of aggregation functions that simultaneously generalize weighted means and OWA operators.
\end{abstract}

Keywords: SUOWA operators, orness, weighted means, OWA operators, Choquet integral.

\section{Introduction}

Aggregation information is a usual activity in many research fields. Weighted means and ordered weighted averaging (OWA) operators (Yager, 1988) are wellknown functions widely used for this task. Both classes of functions are defined by means of weighting vectors, but their behavior is quite different: Weighted means allow to weight each information source in relation to their reliability while OWA operators allow to weight the values according to their ordering. Nevertheless, there are situations where both weightings are necessary (see, for instance, Torra, 1997, 2001; Llamazares, 2015a,b,c; and the references therein).

Different aggregation functions have appeared in the literature to deal with this kind of problems. A usual approach is to consider families of functions parametrized by two weighting vectors, one for the weighted mean and the other one for the OWA type aggregation, that generalize weighted means and OWA operators in the following sense: A weighted mean (or a OWA operator) is obtained when the other weighting vector has a "neutral" behavior; that is, it is $(1 / n, \ldots, 1 / n)$ (see Llamazares, 2013, for an analysis of some functions that gen- 
eralize the weighted means and the OWA operators in this sense). Two of the solutions having better properties are the weighted OWA (WOWA) operator (Torra, 1997 ) and the semi-uninorm based ordered weighted averaging (SUOWA) operator (Llamazares, 2015a).

An important issue in the field of aggregation functions is the behavior of compensative operators regarding the minimum and the maximum. For dealing with this task, the notion of orness was introduced for the case of the root-mean-powers (Dujmović, 1974) and, in an independent way, for OWA operators (Yager, 1988). Later, it has been generalized by using the notion of average value (Marichal, 1998). In this paper we analyze some specific cases of SUOWA operators with respect to this indicator and show how to get a SUOWA operator with a previously fixed degree of orness.

The paper is organized as follows. In Section 2 we recall some basic properties of aggregation functions and the concepts of semi-uninorms and uninorms. Section 3 is devoted to Choquet integral, including some of the most interesting particular cases: weighted means, OWA operators and SUOWA operators. In Section 4 we give some properties of SUOWA operators related to their orness, and illustrate how to obtain a SUOWA operator having a particular degree of orness. Finally, some concluding remarks are provided in Section 5.

\section{Preliminaries}

Throughout the paper we will use the following notation: $N=\{1, \ldots, n\}$; given $A \subseteq N,|A|$ denotes the cardinality of $A$; vectors are denoted in bold and $\boldsymbol{\eta}$ denotes the tuple $(1 / n, \ldots, 1 / n) \in \mathbb{R}^{n}$. We write $\boldsymbol{x} \geq \boldsymbol{y}$ if $x_{i} \geq y_{i}$ for all $i \in N$. For a vector $\boldsymbol{x} \in \mathbb{R}^{n},[\cdot]$ and $(\cdot)$ denote permutations such that $x_{[1]} \geq \cdots \geq x_{[n]}$ and $x_{(1)} \leq \cdots \leq x_{(n)}$.

Some well known properties of aggregation functions are the following.

Definition 1. Let $F: \mathbb{R}^{n} \rightarrow \mathbb{R}$ be a function.

1. $F$ is symmetric if $F\left(x_{\sigma(1)}, \ldots, x_{\sigma(n)}\right)=F\left(x_{1}, \ldots, x_{n}\right)$ for all $\boldsymbol{x} \in \mathbb{R}^{n}$ and for all permutation $\sigma$ of $N$.

2. $\quad F$ is monotonic if $\boldsymbol{x} \geq \boldsymbol{y}$ implies $F(\boldsymbol{x}) \geq F(\boldsymbol{y})$ for all $\boldsymbol{x}, \boldsymbol{y} \in \mathbb{R}^{n}$.

3. $F$ is idempotent if $F(x, \ldots, x)=x$ for all $x \in \mathbb{R}$.

4. $F$ is compensative (or internal) if $\min (\boldsymbol{x}) \leq F(\boldsymbol{x}) \leq \max (\boldsymbol{x})$ for all $x \in \mathbb{R}^{n}$.

5. $F$ is homogeneous of degree 1 (or ratio scale invariant) if $F(r \boldsymbol{x})=r F(\boldsymbol{x})$ for all $\boldsymbol{x} \in \mathbb{R}^{n}$ and for all $r>0$.

A class of necessary functions in the definition of SUOWA operators are semiuninorms (Liu, 2012). These functions are monotonic and have a neutral element in the interval $[0,1]$. They were introduced as a generalization of uninorms, which, 
in turn, were proposed as a generalization of t-norms and t-conorms (Yager and Rybalov, 1996).

Definition 2. Let $U:[0,1]^{2} \rightarrow[0,1]$.

1. $U$ is is a semi-uninorm if it is monotonic and possesses a neutral element $e \in[0,1]$ (for all $x \in[0,1], U(e, x)=U(x, e)=x$ ).

2. $U$ is a uninorm if it is a symmetric and associative (for all $x, y, z \in[0,1]$, $U(x, U(y, z))=U(U(x, y), z))$ semi-uninorm.

We denote by $\mathcal{U}^{e}$ (respectively, $\mathcal{U}_{\mathrm{i}}^{e}$ ) the set of semi-uninorms (respectively, idempotent semi-uninorms) with neutral element $e \in[0,1]$.

SUOWA operators are defined by using semi-uninorms with neutral element $1 / n$. Moreover, they have to belong to the following subset (Llamazares, 2015a):

$$
\tilde{u}^{1 / n}=\left\{U \in \mathcal{U}^{1 / n} \mid U(1 / k, 1 / k) \leq 1 / k \text { for all } k \in N\right\} .
$$

Obviously $\mathcal{U}_{\mathrm{i}}^{1 / n} \subseteq \tilde{\mathcal{U}}^{1 / n}$. Notice that the smallest and the largest elements of $u_{\mathrm{i}}^{1 / n}$ are, respectively, the following uninorms (Yager and Rybalov, 1996):

$$
\begin{aligned}
U_{\min }(x, y) & = \begin{cases}\max (x, y), & \text { if }(x, y) \in[1 / n, 1]^{2}, \\
\min (x, y), & \text { otherwise, }\end{cases} \\
U_{\max }(x, y) & = \begin{cases}\min (x, y), & \text { if }(x, y) \in[0,1 / n]^{2}, \\
\max (x, y), & \text { otherwise. }\end{cases}
\end{aligned}
$$

\section{Choquet integral}

The notion of Choquet integral is based on that of capacity (Choquet, 1953; Murofushi and Sugeno, 1991). The concept of capacity resembles that of probability measure but in the definition of the former additivity is replaced by monotonicity (see also fuzzy measures in Sugeno, 1974). A game is then a generalization of a capacity where the monotonicity is no longer required.

\section{Definition 3.}

1. A game $v$ on $N$ is a set function $v: 2^{N} \rightarrow \mathbb{R}$ satisfying $v(\varnothing)=0$.

2. A capacity (or fuzzy measure) $\mu$ on $N$ is a game on $N$ satisfying $\mu(A) \leq$ $\mu(B)$ whenever $A \subseteq B$. In particular, it follows that $\mu: 2^{N} \rightarrow[0, \infty)$. A capacity $\mu$ is said to be normalized if $\mu(N)=1$.

A straightforward way to get a capacity from a game is to consider the monotonic cover of the game (Maschler and Peleg, 1967; Maschler et al., 1971).

Definition 4. Let $v$ be a game on $N$. The monotonic cover of $v$ is the set function $\hat{v}$ given by

$$
\hat{v}(A)=\max _{B \subseteq A} v(B) .
$$


Some basic properties of $\hat{v}$ are given in the sequel.

Remark 1. Let $v$ be a game on $N$. Then:

1. $\hat{v}$ is a capacity on $N$.

2. If $v$ is a capacity on $N$, then $\hat{v}=v$.

3. If $v(A) \leq 1$ for all $A \subseteq N$ and $v(N)=1$, then $\hat{v}$ is a normalized capacity on $N$.

Although the Choquet integral is usually defined as a functional (see, for instance, Choquet, 1953; Murofushi and Sugeno, 1991), in this paper we consider the Choquet integral as an aggregation function over $\mathbb{R}^{n}$ (see, for instance, Grabisch et al., 2009, p. 181).

Definition 5. Let $\mu$ be a capacity on $N$. The Choquet integral with respect to $\mu$ is the function $\mathcal{C}_{\mu}: \mathbb{R}^{n} \rightarrow \mathbb{R}$ given by

$$
\mathcal{C}_{\mu}(\boldsymbol{x})=\sum_{i=1}^{n} \mu\left(B_{(i)}\right)\left(x_{(i)}-x_{(i-1)}\right),
$$

where $B_{(i)}=\{(i), \ldots,(n)\}$, and we use the convention $x_{(0)}=0$.

It is worth noting that Choquet integral-based operators possess several properties which are useful in certain information aggregation contexts (Grabisch et al., 2009, p. 193 and p. 196).

Remark 2. Let $\mu$ be a capacity on $N$. Then $\mathcal{C}_{\mu}$ is continuous, monotonic and homogeneous of degree 1. Moreover, it is idempotent and compensative when $\mu$ is a normalized capacity.

Choquet integral can also be represented by using a decreasing sequences of values (see, for instance, Torra, 1998; Llamazares, 2015a):

$$
\mathcal{C}_{\mu}(\boldsymbol{x})=\sum_{i=1}^{n} \mu\left(A_{[i]}\right)\left(x_{[i]}-x_{[i+1]}\right),
$$

where $A_{[i]}=\{[1], \ldots,[i]\}$, and we use the convention $x_{[n+1]}=0$.

From the previous expression, it is straightforward to show explicitly the weights of the values $x_{[i]}$ by representing the Choquet integral as follows:

$$
\mathcal{C}_{\mu}(\boldsymbol{x})=\sum_{i=1}^{n}\left(\mu\left(A_{[i]}\right)-\mu\left(A_{[i-1]}\right)\right) x_{[i]},
$$

where we use the convention $A_{[0]}=\varnothing$. 


\subsection{Weighted means and OWA operators}

Weighted means and OWA operators (Yager, 1988) are well-known functions in the theory of aggregation operators. Both classes of functions are defined in terms of weight distributions that add up to 1 .

Definition 6. A vector $\boldsymbol{q} \in \mathbb{R}^{n}$ is a weighting vector if $\boldsymbol{q} \in[0,1]^{n}$ and $\sum_{i=1}^{n} q_{i}=$ 1.

Definition 7. Let $\boldsymbol{p}$ be a weighting vector. The weighted mean associated with $\boldsymbol{p}$ is the function $M_{\boldsymbol{p}}: \mathbb{R}^{n} \rightarrow \mathbb{R}$ given by

$$
M_{\boldsymbol{p}}(\boldsymbol{x})=\sum_{i=1}^{n} p_{i} x_{i}
$$

Definition 8. Let $\boldsymbol{w}$ be a weighting vector. The OWA operator associated with $\boldsymbol{w}$ is the function $O_{\boldsymbol{w}}: \mathbb{R}^{n} \rightarrow \mathbb{R}$ given by

$$
O_{w}(\boldsymbol{x})=\sum_{i=1}^{n} w_{i} x_{[i]} .
$$

It is well known that weighted means and OWA operators are specific cases of Choquet integral (Fodor et al., 1995; Grabisch, 1995a,b; Llamazares, 2015a).

\section{Remark 3.}

1. If $\boldsymbol{p}$ is a weighting vector, then $M_{\boldsymbol{p}}$ is the Choquet integral with respect to the normalized capacity $\mu_{\boldsymbol{p}}(A)=\sum_{i \in A} p_{i}$.

2. If $\boldsymbol{w}$ is a weighting vector, then $O_{w}$ is the Choquet integral with respect to the normalized capacity $\mu_{|\boldsymbol{w}|}(A)=\sum_{i=1}^{|A|} w_{i}$.

So, according to Remark 2, weighted means and OWA operators are continuous, monotonic, idempotent, compensative and homogeneous of degree 1. Moreover, in the case of OWA operators, given that the values of the variables are previously ordered in a decreasing way, they are also symmetric.

\subsection{SUOWA operators}

SUOWA operators (Llamazares, 2015a) were introduced in order to consider situations where both the importance of information sources and the importance of values had to be taken into account. These functions are Choquet integral-based operators where their capacities are the monotonic cover of specific games. These games are defined by using semi-uninorms with neutral element $1 / n$ and the values of the capacities associated with the weighted means and the OWA operators. 
To be specific, the games from which SUOWA operators are built are defined as follows.

Definition 9. Let $\boldsymbol{p}$ and $\boldsymbol{w}$ be two weighting vectors and let $U \in \tilde{U}^{1 / n}$.

1. The game associated with $\boldsymbol{p}, \boldsymbol{w}$ and $U$ is the set function $v_{p, \boldsymbol{w}}^{U}: 2^{N} \rightarrow \mathbb{R}$ defined by

$$
\begin{aligned}
& \qquad v_{p, w}^{U}(A)=|A| U\left(\frac{\mu_{p}(A)}{|A|}, \frac{\mu_{|w|}(A)}{|A|}\right) \\
& \text { if } A \neq \emptyset \text {, and } v_{p, w}^{U}(\varnothing)=0 \text {. }
\end{aligned}
$$

2. $\hat{v}_{p, w}^{U}$, the monotonic cover of the game $v_{p, w}^{U}$, will be called the capacity associated with $\boldsymbol{p}, \boldsymbol{w}$ and $U$.

Definition 10. Let $\boldsymbol{p}$ and $\boldsymbol{w}$ be two weighting vectors and let $U \in \tilde{U}^{1 / n}$. The SUOWA operator associated with $\boldsymbol{p}, \boldsymbol{w}$ and $U$ is the function $S_{\boldsymbol{p}, \boldsymbol{w}}^{U}: \mathbb{R}^{n} \rightarrow \mathbb{R}$ given by

$$
S_{\boldsymbol{p}, \boldsymbol{w}}^{U}(\boldsymbol{x})=\sum_{i=1}^{n} s_{i} x_{[i]},
$$

where $s_{i}=\hat{v}_{p, w}^{U}\left(A_{[i]}\right)-\hat{v}_{p, \boldsymbol{w}}^{U}\left(A_{[i-1]}\right)$ for all $i \in N, \hat{v}_{\boldsymbol{p}, \boldsymbol{w}}^{U}$ is the capacity associated with $\boldsymbol{p}, \boldsymbol{w}$ and $U$, and $A_{[i]}=\{[1], \ldots,[i]\}$ (with the convention that $A_{[0]}=\varnothing$ ).

According to expression (1), the SUOWA operator associated with $\boldsymbol{p}, \boldsymbol{w}$ and $U$ can also be written as

$$
S_{\boldsymbol{p}, \boldsymbol{w}}^{U}(\boldsymbol{x})=\sum_{i=1}^{n} \hat{v}_{\boldsymbol{p}, \boldsymbol{w}}^{U}\left(A_{[i]}\right)\left(x_{[i]}-x_{[i+1]}\right) .
$$

By the choice of $\hat{v}_{p, w}^{U}$ we have $S_{p, \eta}^{U}=M_{p}$ and $S_{\eta, w}^{U}=O_{\boldsymbol{w}}$ for any $U \in \tilde{U}^{1 / n}$ Moreover, by Remark 2 and given that $\hat{v}_{p, w}^{U}$ is a normalized capacity, SUOWA operators are continuous, monotonic, idempotent, compensative and homogeneous of degree 1.

\section{Orness measures}

An important concept to measure the degree to which the aggregation is disjunctive (i.e. it is like an or operation) is the notion of orness. This concept was initially introduced for the case of the root-mean-powers (Dujmović, 1974) and, in an independent way, for OWA operators (Yager, 1988). Afterwards, Marichal (1998) derived an orness measure for the Choquet integral by using the notion of average value. 
Definition 11. Let $\mu$ be a capacity on $N$.

1. The average value of $\mathcal{C}_{\mu}$ is defined by

$$
E\left(\mathcal{C}_{\mu}\right)=\int_{[0,1]^{n}} \mathcal{C}_{\mu}(\boldsymbol{x}) d \boldsymbol{x} .
$$

2. The orness value of $\mathcal{C}_{\mu}$ is defined by

$$
\operatorname{orness}\left(\mathcal{C}_{\mu}\right)=\frac{E\left(\mathcal{C}_{\mu}\right)-E(\min )}{E(\max )-E(\min )}
$$

The orness of $\mathcal{C}_{\mu}$ can be written in terms of the capacity $\mu$ (Marichal, 2004):

$$
\operatorname{orness}\left(\mathcal{C}_{\mu}\right)=\frac{1}{n-1} \sum_{t=1}^{n-1} \frac{1}{\left(\begin{array}{c}
n \\
t
\end{array}\right)} \sum_{\substack{T \subseteq N \\
|T|=t}} \mu(T) .
$$

The degree of orness is known for some Choquet integral-based operators (Marichal, 2004). In fact, in the particular cases of weighted means and OWA operators we get the following values:

$$
\operatorname{orness}\left(M_{\boldsymbol{p}}\right)=\frac{1}{2}, \quad \operatorname{orness}\left(O_{\boldsymbol{w}}\right)=\frac{1}{n-1} \sum_{i=1}^{n}(n-i) w_{i} .
$$

However, in the vast majority of cases, the calculation of this value through a closed expression does not seem an easy task (Torra, 2001). In the case of SUOWA operators, we can get a very interesting result when we consider a semiuninorm obtained as a convex combination of two semi-uninorms satisfying that the games associated with them are capacities. In this case, the orness of the SUOWA operator associated with this new semi-uninorm can be gotten through the same convex combination of the orness of the SUOWA operators associated with the former semi-uninorms. This result, together with others concerning the game and the SUOWA operator associated with this new semi-uninorm, are collected in the following theorem.

Theorem 1. Let $\boldsymbol{p}$ and $\boldsymbol{w}$ be two weighting vectors, let $U_{1}, U_{2} \in \tilde{U}^{1 / n}$ such that $v_{\boldsymbol{p}, \boldsymbol{w}}^{U_{1}}$ and $v_{\boldsymbol{p}, \boldsymbol{w}}^{U_{2}}$ be capacities, let $\lambda \in[0,1]$, and let $U=\lambda U_{1}+(1-\lambda) U_{2}$. Then:

1. $U \in \tilde{\mathcal{U}}^{1 / n}$ and if $U_{1}, U_{2} \in \mathcal{U}_{\mathrm{i}}^{1 / n}$ then $U \in \mathcal{U}_{\mathrm{i}}^{1 / n}$.

2. $v_{\boldsymbol{p}, \boldsymbol{w}}^{U}(A)=\lambda v_{\boldsymbol{p}, \boldsymbol{w}}^{U_{1}}(A)+(1-\lambda) v_{\boldsymbol{p}, \boldsymbol{w}}^{U_{2}}(A)$ for any subset $A$ of $N$.

3. $v_{p, w}^{U}$ is a normalized capacity on $N$.

4. $S_{\boldsymbol{p}, \boldsymbol{w}}^{U}(\boldsymbol{x})=\lambda S_{\boldsymbol{p}, \boldsymbol{w}}^{U_{1}}(\boldsymbol{x})+(1-\lambda) S_{\boldsymbol{p}, \boldsymbol{w}}^{U_{2}}(\boldsymbol{x})$ for all $\boldsymbol{x} \in \mathbb{R}^{n}$.

5. $\operatorname{orness}\left(S_{\boldsymbol{p}, \boldsymbol{w}}^{U}\right)=\lambda \operatorname{orness}\left(S_{\boldsymbol{p}, \boldsymbol{w}}^{U_{1}}\right)+(1-\lambda) \operatorname{orness}\left(S_{\boldsymbol{p}, \boldsymbol{w}}^{U_{2}}\right)$.

Among the great variety of semi-uninorms belonging to $\tilde{u}^{1 / n}$ that could be chosen to generate a SUOWA operator, idempotent semi-uninorms are of specific 
interest owing to their notable properties (Llamazares, 2015a). Notice that, since any idempotent semi-uninorm is located between $U_{\min }$ and $U_{\max }$, we straightforwardly obtain the following results.

Remark 4. Let $\boldsymbol{p}$ and $\boldsymbol{w}$ be two weighting vectors, and $U \in \mathcal{U}_{\mathrm{i}}^{1 / n}$. Then:

1. $S_{\boldsymbol{p}, \boldsymbol{w}}^{U_{\min }}(\boldsymbol{x}) \leq S_{\boldsymbol{p}, \boldsymbol{w}}^{U}(\boldsymbol{x}) \leq S_{\boldsymbol{p}, \boldsymbol{w}}^{U_{\max }}(\boldsymbol{x})$ for all $\boldsymbol{x} \in \mathbb{R}^{n}$.

2. $\operatorname{orness}\left(S_{p, w}^{U_{\min }}\right) \leq \operatorname{orness}\left(S_{p, w}^{U}\right) \leq \operatorname{orness}\left(S_{p, w}^{U_{\max }}\right)$.

Given that SUOWA operators generated from idempotent semi-uninorms are located between those obtained from the uninorms $U_{\min }$ and $U_{\max }$, next we establish some results on the games and the SUOWA operators associated with these uninorms when the weighting vector $\boldsymbol{w}$ is an increasing (in the case of considering $U_{\min }$ ) or decreasing (in the case of considering $U_{\max }$ ) sequence of weights.

Theorem 2. Let $\boldsymbol{w}$ be a weighting vector such that $w_{1} \leq w_{2} \leq \cdots \leq w_{n}$ and $\boldsymbol{w} \neq \boldsymbol{\eta}$. Then, for all weighting vector $\boldsymbol{p}$, we have

1. $v_{p, w}^{U_{\min }}$ is a normalized capacity on $N$.

2. $v_{p, \boldsymbol{w}}^{U_{\min }}(A)=\min \left(\mu_{\boldsymbol{p}}(A), \mu_{|\boldsymbol{w}|}(A)\right)$ for all $A \subseteq N$.

3. $S_{\boldsymbol{p}, \boldsymbol{w}}^{U_{\min }}(\boldsymbol{x}) \leq \min \left(M_{\boldsymbol{p}}(\boldsymbol{x}), O_{\boldsymbol{w}}(\boldsymbol{x})\right)$ for all $\boldsymbol{x} \in \mathbb{R}^{n}$.

4. $\operatorname{orness}\left(S_{p, w}^{U_{\min }}\right) \leq \operatorname{orness}\left(O_{w}\right) \leq 0.5$.

Similar results can be established for the uninorm $U_{\max }$.

Theorem 3. Let $\boldsymbol{w}$ be a weighting vector such that $w_{1} \geq w_{2} \geq \cdots \geq w_{n}$ and $\boldsymbol{w} \neq \boldsymbol{\eta}$. Then, for all weighting vector $\boldsymbol{p}$, we get

1. $v_{p, w}^{U_{\max }}$ is a normalized capacity on $N$.

2. $v_{p, w}^{U_{\max }}(A)=\max \left(\mu_{p}(A), \mu_{|w|}(A)\right)$ for all $A \subseteq N$.

3. $S_{\boldsymbol{p}, \boldsymbol{w}}^{U_{\max }}(\boldsymbol{x}) \geq \max \left(M_{\boldsymbol{p}}(\boldsymbol{x}), O_{\boldsymbol{w}}(\boldsymbol{x})\right)$ for all $\boldsymbol{x} \in \mathbb{R}^{n}$.

4. orness $\left(S_{p, w}^{U_{\max }}\right) \geq \operatorname{orness}\left(O_{w}\right) \geq 0.5$.

In the sequel, and using some of the previous results, we illustrate how we can get a SUOWA operator having a particular degree of orness.

Example 1. Let us to consider the weighting vectors $\boldsymbol{p}=(0.1,0.4,0.4,0.1)$ and $\boldsymbol{w}=(0.3,0.3,0.2,0.2)$, and the idempotent uninorms $U_{\min }$ and $U_{\max }$. In the case of $U_{\max }$, the first item of Theorem 3 guarantees that $v_{p, w}^{U_{\max }}$ is a capacity. As we can see in Table 1, this is also the case when we consider the uninorm $U_{\min }$.

According to expressions (2) and (3), we have

$$
\operatorname{orness}\left(O_{w}\right)=1.7 / 3, \operatorname{orness}\left(S_{p, w}^{U_{\min }}\right)=4.7 / 9, \quad \operatorname{orness}\left(S_{p, w}^{U_{\max }}\right)=5.5 / 9 .
$$

And by the second item of Remark 4, for any idempotent semi-uninorm $U$ we get

$$
4.7 / 9 \leq \operatorname{orness}\left(S_{p, w}^{U}\right) \leq 5.5 / 9 \text {. }
$$


Table 1 Capacities associated with $U_{\min }$ and $U_{\max }$.

\begin{tabular}{ccc}
\hline Set & $v_{\boldsymbol{p}, \boldsymbol{w}}^{U_{\min }}$ & $v_{\boldsymbol{p}, \boldsymbol{w}}^{U_{\max }}$ \\
\hline$\{1\}$ & 0.1 & 0.3 \\
$\{2\}$ & 0.4 & 0.4 \\
$\{3\}$ & 0.4 & 0.4 \\
$\{4\}$ & 0.1 & 0.3 \\
$\{1,2\}$ & 0.6 & 0.6 \\
$\{1,3\}$ & 0.6 & 0.6 \\
$\{1,4\}$ & 0.2 & 0.6 \\
$\{2,3\}$ & 0.8 & 0.8 \\
$\{2,4\}$ & 0.6 & 0.6 \\
$\{3,4\}$ & 0.6 & 0.6 \\
$\{1,2,3\}$ & 0.9 & 0.9 \\
$\{1,2,4\}$ & 0.6 & 0.8 \\
$\{1,3,4\}$ & 0.6 & 0.8 \\
$\{2,3,4\}$ & 0.9 & 0.9 \\
$N$ & 1 & 1 \\
\hline
\end{tabular}

Now, by the fifth item of Theorem 1, we can easily obtain an idempotent semiuninorm that allows us to get a SUOWA operator having a particular degree of orness in the range $4.7 / 9$ to $5.5 / 9$. For instance, if we look for an idempotent semiuninorm $U$ such that orness $\left(S_{p, w}^{U}\right)=\operatorname{orness}\left(O_{w}\right)=1.7 / 3$, then, since $1.7 / 3=$ $0.5 \cdot 4.7 / 9+0.5 \cdot 5.5 / 9$, it is sufficient to consider $U_{\mathrm{am}}=0.5 U_{\min }+0.5 U_{\max }$, that is, the idempotent semi-uninorm obtained through the arithmetic mean:

$$
U_{\mathrm{am}}(x, y)=\left\{\begin{array}{cl}
\min (x, y), & \text { if }(x, y) \in[0,0.25]^{2}, \\
\max (x, y), & \text { if }(x, y) \in[0.25,1]^{2} \backslash\{(0.25,0.25)\}, \\
(x+y) / 2, & \text { otherwise. }
\end{array}\right.
$$

\section{Conclusion}

SUOWA operators have been introduced recently for dealing with situations where combining values by using both a weighted mean and a OWA type aggregation is necessary. Given that they are Choquet integral-based operators with respect to normalized capacities, they have some natural properties such as continuity, monotonicity, idempotency, compensativeness and homogeneity of degree 1 . For this reason, it seems interesting to analyze their behavior through indicators proposed in the literature. In this paper we have investigated the orness of some specific cases of SUOWA operators and illustrated how to obtain a SUOWA operator with a given degree of orness. 
Acknowledgments. The partial financial support from the Ministerio de Economía y Competitividad (Project ECO2012-32178) and the Junta de Castilla y León (Consejería de Educación, Project VA066U13) is gratefully acknowledged.

\section{References}

Choquet, G.: Theory of capacities. Annales de l'Institut Fourier 5, 131-295 (1953).

Dujmović, J.J.: Weighted conjunctive and disjunctive means and their application in system evaluation. J. Univ. Belgrade, EE Dept., Ser. Math. and Phys. 483, 147-158 (1974).

Fodor, J., Marichal, J.L. and Roubens, M.: Characterization of the ordered weighted averaging operators. IEEE Transactions on Fuzzy Systems 3, 236-240 (1995).

Grabisch, M.: Fuzzy integral in multicriteria decision making. Fuzzy Sets and Systems 69, 279298 (1995a).

Grabisch, M.: On equivalence classes of fuzzy connectives - the case of fuzzy integrals. IEEE Transactions on Fuzzy Systems 3, 96-109 (1995b).

Grabisch, M., Marichal, J., Mesiar, R. and Pap, E.: Aggregation Functions. Cambridge University Press, Cambridge (2009).

Liu, H.W.: Semi-uninorms and implications on a complete lattice. Fuzzy Sets and Systems 191, 72-82 (2012)

Llamazares, B.: An analysis of some functions that generalizes weighted means and OWA operators. International Journal of Intelligent Systems 28, 380-393 (2013).

Llamazares, B.: Constructing Choquet integral-based operators that generalize weighted means and OWA operators. Information Fusion 23, 131-138 (2015a).

Llamazares, B.: SUOWA operators: Constructing semi-uninorms and analyzing specific cases. Fuzzy Sets and Systems, forthcoming (2015b).

Llamazares, B.: A study of SUOWA operators in two dimensions. Mathematical Problems in Engineering, forthcoming (2015c).

Marichal, J.L.: Aggregation Operators for Multicriteria Decision Aid. Ph.D. thesis, University of Liège (1998).

Marichal, J.L.: Tolerant or intolerant character of interacting criteria in aggregation by the Choquet integral. European Journal of Operational Research 155, 771-791 (2004).

Maschler, M. and Peleg, B.: The structure of the kernel of a cooperative game. SIAM Journal on Applied Mathematics 15, 569-604 (1967).

Maschler, M., Peleg, B. and Shapley, L.S.: The kernel and bargaining set for convex games. International Journal of Game Theory 1, 73-93 (1971).

Murofushi, T. and Sugeno, M.: A theory of fuzzy measures. Representation, the Choquet integral and null sets. Journal of Mathematical Analysis and Applications 159, 532-549 (1991).

Sugeno, M.: Theory of Fuzzy Integrals and its Applications. Ph.D thesis, Tokyo Institute of Technology (1974).

Torra, V.: The weighted OWA operator. International Journal of Intelligent Systems 12, 153-166 (1997).

Torra, V.: On some relationships between the WOWA operator and the Choquet integral. In: Proc. 7th Int. Conf. on Information Processing and Management of Uncertainty in Knowledge-Based Systems (IPMU’98), pp. 818-824, Paris (1998).

Torra, V.: Empirical analysis to determine Weighted OWA orness. In: Proc. 4th Int. Conf. on Information Fusion (FUSION 2001), Montreal (2001).

Yager, R.R.: On ordered weighted averaging operators in multicriteria decision making. IEEE Transactions on Systems, Man, and Cybernetics 18, 183-190 (1988).

Yager, R.R. and Rybalov, A.: Uninorm aggregation operators. Fuzzy Sets and Systems 80, 111120 (1996) 